\title{
Una nueva especie de Eupithecia Curtis (Lepidoptera: Geometridae) del extremo norte de Chile
}

\author{
A new species of Eupithecia Curtis (Lepidoptera: Geometridae) from northernmost Chile
}

\author{
HÉCTOR A. VARGAS ${ }^{1} \&$ LUIS E. PARRA ${ }^{2}$ \\ ${ }^{1}$ Laboratorio de Entomología, Facultad de Agronomía, Universidad de Tarapacá, Casilla 6-D, Arica, Chile; \\ e-mail: havargas@uta.cl \\ ${ }^{2}$ Departamento de Zoología, Facultad de Ciencias Naturales y Oceanográficas, Universidad de Concepción, Casilla 160-C, \\ Concepción, Chile; e-mail: luparra@udec.cl
}

\begin{abstract}
RESUMEN
Se presenta la descripción de una nueva especie de Eupithecia (Lepidoptera: Geometridae). Su distribución comprende tres valles del extremo norte de Chile: Azapa, Chaca y Camarones. Se describen e ilustran los adultos, incluyendo la genitalia de ambos sexos, y el segmento terminal de la pupa de la hembra. Las larvas se alimentan de inflorescencias de Acacia macracantha y Prosopis tamarugo (Fabaceae).
\end{abstract}

Palabras clave: taxonomía, Larentiinae, E. yubitzae sp. nov., Fabaceae.

\begin{abstract}
The description of a new Eupithecia species (Lepidoptera: Geometridae) is presented. Its distribution comprises three valleys in northernmost Chile: Azapa, Chaca and Camarones. The habitus of adult, the genitalia of both sexes, and the last segment of the female pupa are described and illustrated. Larvae feed on inflorescence of Acacia macracantha and Prosopis tamarugo (Fabaceae).
\end{abstract}

Key words: taxonomy, Larentiinae, E. yubitzae sp. nov., Fabaceae.

\section{INTRODUCCIÓN}

El Neotrópico reúne aproximadamente al $38 \%$ de las especies conocidas de Geometridae (Heppner 1991). En Chile esta familia se encuentra representada por más de 450 especies distribuidas en las subfamilias Archierinae, Ennominae, Geometrinae y Larentiinae (Parra 1995).

Eupithecia Curtis es uno de los géneros más diversos de la subfamilia Larentiinae con más de 1.300 especies descritas (Scoble 1999, Parra \& Ibarra-Vidal 2002), y es probablemente el género más ampliamente distribuido. Se encuentra bien representado en el Neotrópico por 352 especies; sin embargo, es escaso en Australia con solo dos especies y está ausente de Nueva Zelandia (Herbulot 2001).

Varios intentos han sido hechos para dividir este género en grupos menores o subgéneros, pero es un taxón muy homogéneo y los elementos que se han utilizado para su diferenciación son caracteres de poca importancia (Rindge 1987, Herbulot 2001). Las distintas especies de Eupithecia se distinguen difícilmente una de otra, debido a su pe- queño tamaño y a su similar patrón de maculación. Sin embargo, a pesar de estas características externas de los imagos, las armaduras genitales de ambos sexos entregan caracteres específicos que permiten discriminar claramente una especie de otra (Rindge 1987, 1991, Bolte 1990).

Las especies de Eupithecia de Chile fueron revisadas por Rindge (1987), y posteriormente se han efectuado algunas adiciones (Rindge 1991, Parra \& Ibarra-Vidal 2002). Actualmente se han registrado 64 especies para la fauna chilena (Herbolut 2001), muchas de las cuales son endémicas, ya sea del territorio continental o de las Islas de Juan Fernández (Rindge 1987). Por otro lado, la información biológica de las diferentes especies chilenas es escasa, solamente se tienen antecedentes sobre cuatro de ellas: $E$. horismoides Rindge y E. robinsoni Parra \& Ibarra-Vidal, ambas asociadas a Gunneraceae, E. atacama (Vojnits), asociada a especies de Chenopodiaceae, y E. sibylla, cuyas larvas son antófagas sobre Fabaceae (Ibarra-Vidal \& Parra 1993, Parra \& Ibarra-Vidal 2002, Vargas \& Parra 2002, Vargas et al. 2002). 
Mediante muestreos tendientes a determinar la diversidad de lepidópteros asociados a yaro, Acacia macracantha Humb. \& Bonpl. ex Willd. (Fabaceae), en los valles de Azapa y Chaca, se obtuvieron ejemplares adultos criados en inflorescencias de yaro a partir del último estadio larval, y se capturaron adicionalmente ejemplares adultos de la misma especie. El examen de los imagos reveló que corresponden a una nueva especie del género Eupithecia. En el presente trabajo se describe esta nueva especie, cuya distribución actualmente conocida está limitada a tres valles costeros del extremo norte de Chile.

\section{MATERIALES Y MÉTODOS}

El material usado en este estudio proviene de crianzas y capturas realizadas en los valles de Azapa y Chaca, I Región de Chile, y de la Colección Entomológica de la Universidad de Tarapacá (IDEA). Todos los ejemplares fueron comparados con la información publicada (Rindge 1987, 1991, Landry \& Rindge 1995) y con otros ejemplares capturados en el norte de Chile. Puesto que se contó con dos exuvios pupales (ambos hembras), se esquematizó y describió el segmento terminal de dicha estructura, pues presenta una serie de caracteres que pueden ser útiles en la determinación de taxa hasta nivel de grupos de especies o a nivel específico (Bolte 1990). Los ejemplares utilizados en este estudio se encuentran depositados en el Museo de Zoología de la Universidad de Concepción (UCCC), en el Museum d'Histoire Naturelle, Ginebra, Suiza (MHNG) y en la Colección IDEA. La terminología utilizada en la descripción corresponde a la de McGuffin (1988) para la maculación alar, y a Bolte (1990) para la genitalia y el segmento terminal de la pupa.

\section{RESULTADOS}

Eupithecia yubitzae $n . s p$.

Material tipo. Chile: Arica. Holotipo 1 Ỏ: Camarones, Cuya, I Región. 08-04-1993, en Tamarugo, ob. lab. Coll. R. Mendoza (UCCC). Alotipo 1 \& : Camarones, Cuya, I Región. 0804-1993, en Tamarugo, ob. lab. Coll. R. Mendoza (UCCC). Paratipos: $2 \oint^{\circledR}, 1$ : Chaca, I Región, Chile, en flor Yaro, julio 2003, ob. lab. H.A. Vargas coll.; 19: Azapa, K - 20, flor Yaro. 30-12-2001, ob. lab.: 22-01-2002. H.A. Vargas coll. (MHNG); 10ึ: Chaca, I Región, Chile, en flor yaro, julio 2003, ob. lab. H.A. Vargas coll.; 1ㅇ: Azapa, K-12, I Región, Chile, 18-12-2001.
H. A. Vargas, coll; 19: Azapa, K-12, I Región, Chile, 12-09-2001. H. A. Vargas, coll. (IDEA).

Diagnosis. Los imagos de esta especie son geométridos de pequeño tamaño, con el cuerpo y alas cubiertas de escamas de color pardo rojizo claro y algunas escamas grises, negras y blancas. Eupithecia yubitzae se distingue fácilmente de las restantes especies chilenas del género porque el macho presenta el esternito VIII provisto de dos proyecciones posteriores conspicuas esclerosadas, agudas y curvadas, con los ápices dirigidos hacia la línea media a manera de fórceps; la genitalia del macho presenta valvas más anchas en la base y estrechas en la porción distal, el borde dorsal de la valva es curvo antes de alcanzar el tegumen, y la vesica presenta un cornutus conspicuo y numerosos cornuti espiniformes más pequeños; la genitalia de la hembra presenta el corpus bursae notablemente esclerosado, y numerosas espinas cónicas y algunas de forma irregular se disponen sobre la superficie interna del corpus bursae, especialmente concentradas en el área lateroposterior izquierda.

Macho. (Fig. 1) Cabeza con ojos prominentes de contorno circular en vista lateral, provista de escamas pardo rojizas y negras en la frente. Antenas bipectinadas. Tórax y abdomen cubierto de escamas pardo rojizas claras. Ala anterior con superficie dorsal cubierta de escamas de color similar al resto del cuerpo, banda medial negra y fina, banda subterminal blanca y sinuosa, banda terminal negra muy fina, abundantes escamas negras dispuestas en la mitad proximal del área subcostal; superficie ventral más clara que la dorsal, escamas negras subcostales cubren además el margen de la celda discal. Ala posterior con área subcostal prácticamente desprovista de escamas, el resto similar al ala anterior; superficie ventral más clara que la dorsal, cubierta de escamas negras ubicadas en la porción basal del área subcostal. Esternito VIII simétrico; longitud similar a la de las valvas; estrecho en la base; membranoso en sus $2 / 3$ basales; provisto de dos proyecciones posteriores conspicuas esclerosadas, agudas y curvadas, con los ápices dirigidos hacia la línea media a manera de fórceps. Márgenes laterales sinuosos, con una invaginación posterior al margen anterior. Genitalia: (Fig. 2) uncus simple bien diferenciado y esclerosado, base aplanada, ápice curvo, subcilíndrico y aguzado en su extremo distal; valvas más anchas en la base y estrechas en la porción distal, ancho del área distal cerca de 1/6 el ancho basal, ápice distal romo, margen costal curvado antes de alcanzar el tegumen, con los $2 / 3$ distales curvados hacia el dorso, margen ventral con una eva- 


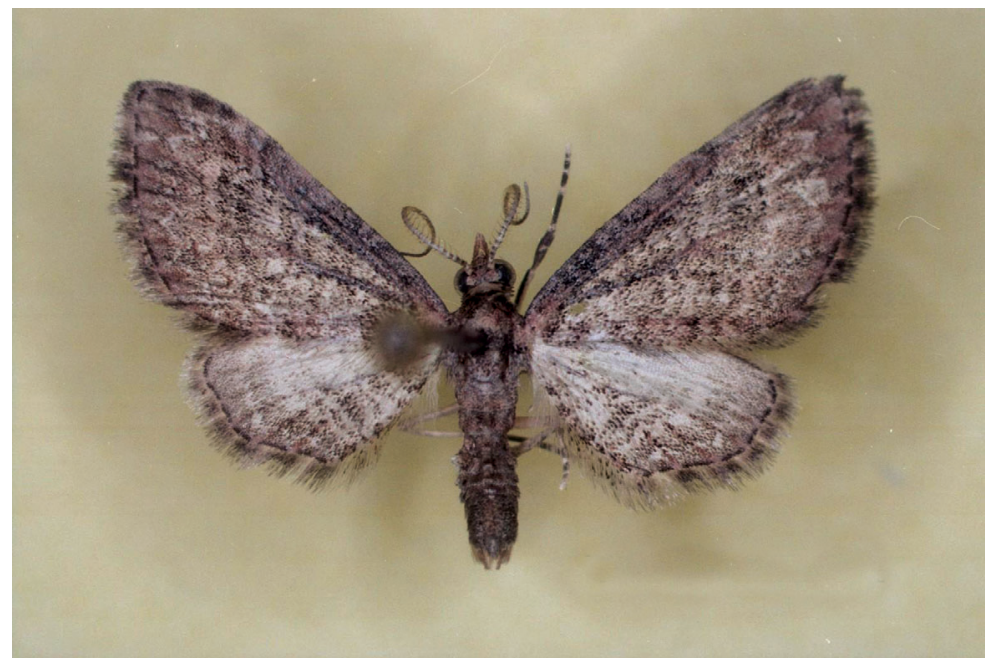

Fig. 1: Imago O̊ de E.yubitzae. Vista dorsal. Escala: $5 \mathrm{~mm}$. ImagoỎ de E.yubitzae. Dorsal view. Scale: $5 \mathrm{~mm}$.

(A)

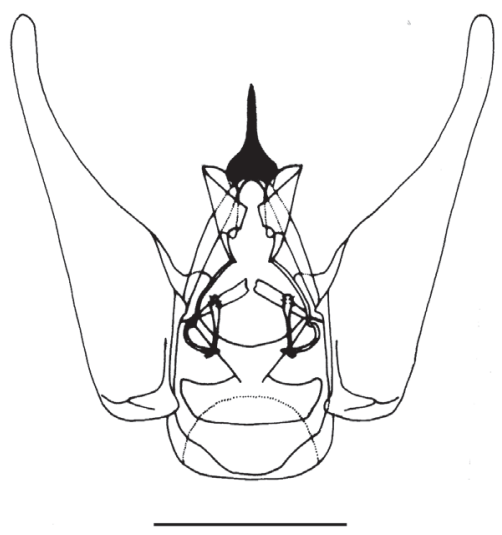

(B)

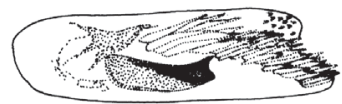

(C)

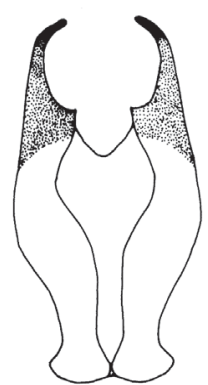

(D)

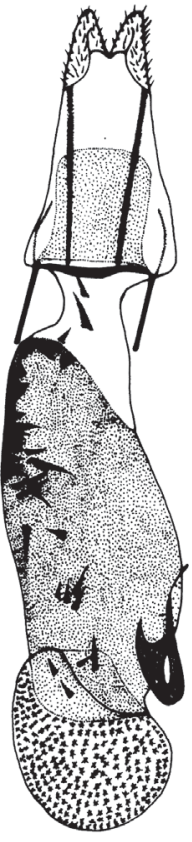

Fig. 2: E. yubitzae. (A) Genitalia del macho en vista ventral; (B) aedeagus en vista lateral; (C) placa ventral del octavo segmento abdominal del macho; (D) genitalia de la hembra en vista ventral. Escalas: $1 \mathrm{~mm}$.

E. yubitzae. (A) Male genitalia in ventral view; (B) Aedeagus in lateral view; (C) ventral plate of the eighth abdominal segment of the male; (D) female genitalia in ventral view. Scales: $1 \mathrm{~mm}$. 
ginación cercana a la base, ligeramente curvo hacia el dorso en su 1/4 distal; región saccular estrecha; anellus compuesto de un área basal subrectangular, cuyo margen distal se presenta ligeramente curvo hacia la base, área distal con aspecto de triángulo invertido, margen distal ampliamente invaginado; papillae digitiformes, levemente dilatadas en el extremo distal, longitud cerca de 3/4 la longitud del uncus, provistas de pequeños pelos ubicados en posición subapical; saccus amplio, de ápice redondeado; aedeagus subcilíndrico, longitud inferior a la de las valvas; vesica armada de una serie de numerosos cornuti cónicos, dispuestos a lo largo de la línea media del aedeagus, un gran cornutus elongado y aplanado, con los márgenes ligeramente curvos y de longitud aproximadamente $1 / 3$ la longitud del eadeagus, se dispone paralelo a la serie de cornuti cónicos; el esclerosamiento de este cornutus es más notorio en su porción distal que en la basal.

Hembra. (Fig. 3) Antenas filiformes. Maculación alar similar a la del macho, pero carece de escamas negras subcostales, tanto en la superficie dorsal como ventral. Genitalia de la hembra: (Fig. 3) apófisis anteriores más de 1/3 la longitud de las apófisis posteriores; lamella postvaginalis muy reducida; ductus bursae membranoso; corpus bursae elongado, fuertemente esclerosado, márgenes sinuosos; numerosas espinas cónicas y algunas de forma irregular se disponen sobre la superficie interna del corpus bursae, especialmente concentradas en el área lateroposterior izquierda; apendix bursae en forma de C, con fuerte curvatura dorsocaudal, conectado ventralmente al corpus bursae en posición subapical derecha; ductus seminalis ubicado en el ápice del appendix bursae; diverticulum membranoso conspicuo subesférico inserto en la región anterior del corpus bursae, provisto de numerosas espinas esteladas de pequeño tamaño.

Etimología. El nombre de esta nueva especie está dedicado a la Sra. Yubitza J. Cortés Jorquera.

Morfología del segmento pupal terminal de la hembra (Fig. 4). Bien diferenciado, área basal, área apical y cremáster claramente distinguibles; ancho de la base menor a la longitud del segmento; cremáster compuesto de ocho setas filiformes esclerosadas, aguzadas y ligeramente curvas en el ápice; seta L1 y D2 ligeramente más gruesas que D1 y SD1; surco dorsal conspicuo y marcadamente sinuoso; constricción medial poco diferenciada; lóbulos laterales conspicuos; se distinguen fácilmente en vista dorsal.

Plantas hospederas. Las larvas de E. yubitzae tienen hábitos antofágicos, y han sido cap- turadas sobre dos especies de leguminosas arbóreas: Acacia macracantha Willd. y Prosopis tamarugo Phil.

Distribución. La presencia de E. yubitzae se conoce solo de la provincia de Arica, en el extremo norte de Chile: valles de Azapa, Chaca y Camarones. Sin embargo, estos datos pueden considerarse simplemente como antecedentes preliminares, puesto que nuevas prospecciones podrían revelar un rango de distribución más amplio.

\section{DISCUSIÓN}

Rindge (1987) dividió el conjunto de especies de Eupithecia de Chile en dos Secciones, para lo cual tomó como base principal las características del segmento VIII del abdomen del macho. La morfología del esternito VIII en $E$. yubitzae permitiría incluirla en la Sección 1. Sin embargo, este es mucho más conspicuo y curvo que en cualquier otra especie chilena; además, la posesión de un elevado número de cornuti en la vesica del macho de E. yubitzae no concuerda con la definición de esta sección, puesto que las demás especies componentes de esta presentan la vesica armada de solo dos piezas esclerosadas. En todo caso, la subdivisión en dos secciones y grupos de Eupithecia realizada por Rindge (1987), es solamente una aproximación inicial que pretende encontrar grupos naturales. Similarmente, Vojnits (1985) realizó un intento para separar Eupithecia en varios géneros distintos; sin embargo, los caracteres diagnósticos utilizados solo representan pequeñas variaciones que no justifican el cambio taxonómico. La evidencia morfológica aportada por las recientes nuevas especies, E. robinsoni (Parra \& Ibarra-Vidal 2002) y la presente proposición, sugieren que estas divisiones probablemente son polifiléticas y que necesariamente un análisis que involucre a todas las especies del género permitiría establecer el verdadero grado de parentesco entre las distintas entidades que lo conforman.

La morfología de la armadura genital del macho y de la hembra de E. yubitzae es similar a la de Eupithecia galapagosata Landry \& Rindge, de las Islas Galápagos. Sin embargo, ambas especies son fácilmente separables sobre la base de estos caracteres: E. yubitzae presenta el borde dorsal de la valva curvo antes de alcanzar el tegumen, mientras que en E. galapagosata es recto; además, el cornutus más grande de la vesica presenta un aspecto distinto en ambas especies. Las hembras pueden ser diferenciadas por el número y disposición de espi- 


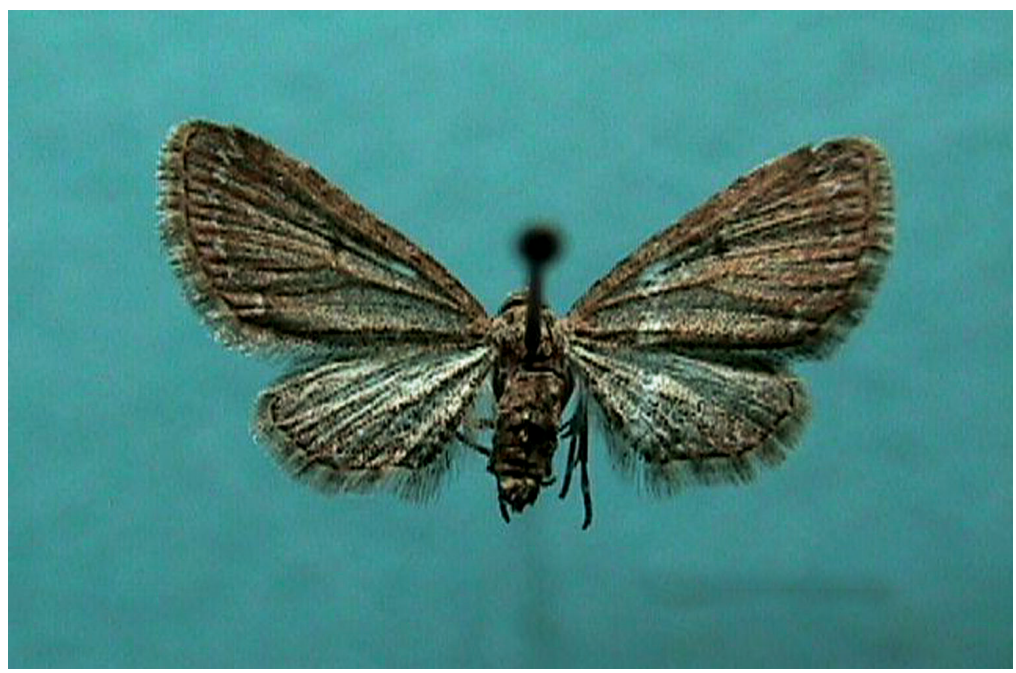

Fig. 3: Imago 9 de E. yubitzae. Vista dorsal. Escala: $5 \mathrm{~mm}$. Imago ${ }_{\text {de E. }}$ yubitzae. Dorsal view. Scale: $5 \mathrm{~mm}$.

(A)
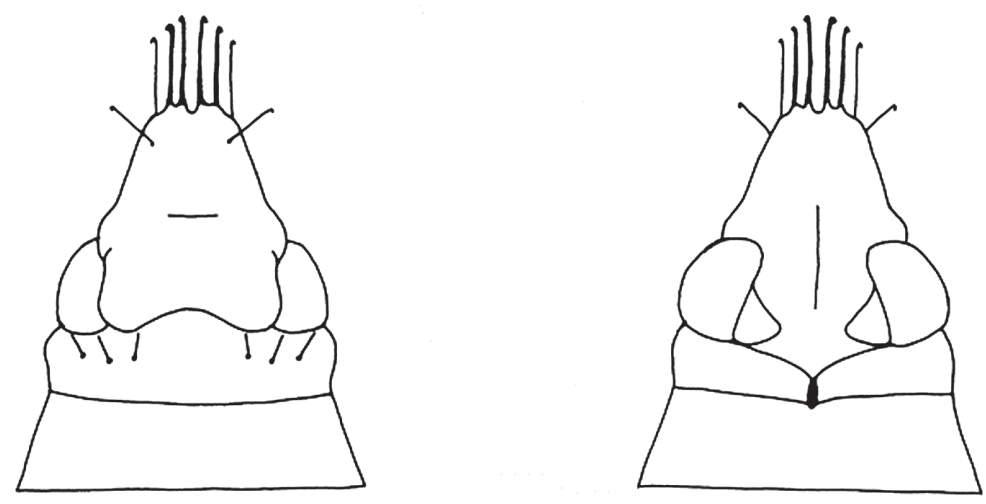

(C)

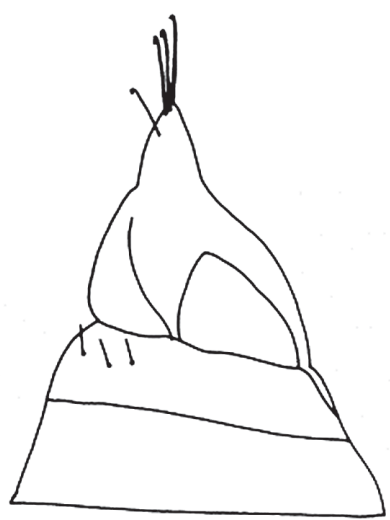

Fig. 4: E. yubitzae. Segmento pupal terminal de la hembra. (A) Vista dorsal; (B) vista lateral; (C) vista ventral. Escala: $1 \mathrm{~mm}$.

E. yubitzae. Female end pupal segment. (A) Dorsal view; (B) lateral view; (C) ventral view. Scale: $1 \mathrm{~mm}$. 
nas en el corpus bursae; además, el corpus bursae en E. yubitzae tiene un aspecto menos curvo que en E. galapagosata, y carece de las estriaciones y de la extensión posterior cercana al ductus bursae presentes en E. galapagosata; el appendix bursae es más angosto y las espinas presentes en el diverticulum bursae son más gruesas en E. yubitzae. Pese a que la apariencia externa de los imagos de E. yubitzae y E. galapagosata es notablemente distinta, la semejanza existente al nivel de las armaduras genitales del macho y hembra de estas dos especies permite suponer que ambas podrían haber derivado de un ancestro común, patrón evolutivo que solamente podrá ser evaluado cuando se realice un estudio que busque las relaciones de parentesco entre las distintas especies de Eupithecia que habitan esta región del mundo.

El género parece estar asociado a una gran variedad de familias de plantas e incluye muchas especies polífagas (Scoble 1999). Los únicos registros de plantas hospederas de especies chilenas del género Eupithecia indican asociaciones oligofágicas con Gunneraceae (E. horismoides, E. robinsoni), Chenopodiaceae (E. atacama) y Fabaceae (E. sibylla) (Ibarra \& Parra 1993, Parra \& Ibarra-Vidal 2002, Vargas et al. 2002, Vargas \& Parra 2002). E. yubitzae corresponde a la segunda especie chilena cuyas larvas son antófagas sobre Fabaceae. La asociación de Eupithecia con Acacia ha sido mencionada previamente para una especie de Sudáfrica y otra de Costa Rica (A. Hausmann, comunicación personal). Lamentablemente, la planta hospedera de E. galapagosata es aún desconocida (Landry \& Rindge 1995).

Eupithecia yubitzae es la especie más septentrional del género en Chile, y esta podría ser la razón de que no exista una gran afinidad con las restantes especies chilenas, sino que con otra Neotropical, como es E. galapagosata.

\section{AGRADECIMIENTOS}

Los autores agradecen al Dr. Bernard Landry, Museum d'Histoire Naturelle, Ginebra, Suiza, y al Dr. Axel Hausmann, Zoologische Staatssammlung, Munich, Alemania, por la revisión del manuscrito y los valiosos comentarios efectuados sobre el mismo; y a Mauricio, Marcelo y Daniel Vargas, por la colaboración en la prospección de larvas. El apoyo financiero para esta investigación fue obtenido de los proyectos DIPOG 9704-03 y 9702-03, Dirección de Investigación y Postgrado, Universidad de Tarapacá (HA Vargas) y 200.113.056-1.0, Dirección de Investigación, Universidad de Concepción (LE Parra).

\section{LITERATURA CITADA}

BOLTE KB (1990) Guide to the geometridae of Canada (Lepidoptera). VI. Subfamily Larentiinae. 1. Revision of the genus Eupithecia. Memoirs of the Entomological Society of Canada 151: 1-253.

IBARRA-VIDAL H \& LE PARRA (1993) Descripción de los estados preimaginales y aspectos de la historia natural de Eupithecia horismoides Rindge 1987 (Lepidoptera: Geometridae), perforador del pecíolo del pangue (Gunnera tinctoria). Revista Chilena de Entomología 20: 35-41.

HEPPNER JB (1991) Faunal regions and the diversity of Lepidoptera. Tropical Lepidoptera 2 (Supplement 1): $1-85$.

HERBULOT C (2001) On Neotropical Eupithecia. En: Proceedings of Forum Herbulot. Spixiana 24: $193-$ 202.

LANDRY B \& FH RINDGE (1995) Additions to the geometridae (Lepidoptera) of the Galápagos Islands, Ecuador, including a new species of Eupithecia. American Museum Novitates 3.118: 1-10.

MCGUFFIN WC (1988) Guide to the geometridae of Canada (Lepidoptera). III, IV, V. Subfamilies Archierinae, Oenochrominae and Geometrinae. Memoirs of The Entomological Society of Canada 145: 1-56.

PARRA LE (1995) Lepidoptera. En: Simonetti JA, MTK Arroyo, AE Sportono \& E Lozada (eds) Diversidad biológica de Chile: 269-279 Comité Nacional de Diversidad Biológica, CONICYT, Santiago, Chile.

PARRA LE \& H IBARRA-VIDAL (2002) A new species of Eupithecia (Lepidoptera: Geometridae) from Juan Fernández Islands. Annals of the Entomological Society of America 95: 9-15.

RINDGE FH (1987) The Eupithecia (Lepidoptera: Geometridae) of Chile. Bulletin of the American Museum of Natural History 186: 269-363.

RINDGE FH (1991) The Eupithecia (Lepidoptera: Geometridae) of Chile. II. American Museum Novitates 3.020: $1-14$

SCOBLE MJ (1999) Geometrid moths of the world: a catalogue (Lepidoptera, Geometridae). CSIRO Publishing, Natural History Museum, London, United Kingdom. 1.016 pp.

VARGAS HA \& LE PARRA (2002) Notas sobre Eupithecia atacama (Vojnits) (Lepidoptera: Geometridae) Idesia 20: 27-33.

VARGAS HA, LE PARRA, HE VARGAS \& DE BOBADILLA (2002) Aspectos biológicos de Eupithecia sybilla Butler 1882 (Lepidoptera: Geometridae) Gayana Zoología (Chile) 66: 103-106.

VOJNITS AM (1985) New South American genera and species of Eupithicini (Lepidoptera: Geometridae). Acta Zoologica Hungarica 31: 405-418. 